\title{
Chaotic Wannier-Bloch resonance states
}

\author{
M. Glück, A. R. Kolovsky ${ }^{\dagger}$ and H. J. Korsch \\ Fachbereich Physik, Universität Kaiserslautern, D-67653 Kaiserslautern, Germany \\ $\dagger$ and L. V. Kirensky Institute of Physics, 660036 Krasnoyarsk, Russia
}

(March 16, 1998)

\begin{abstract}
The Wannier-Bloch resonance states are metastable states of a quantum particle in a space-periodic potential plus a homogeneous field. Here we analyze the states of quantum particle in space- and time-periodic potential. In this case the dynamics of the classical counterpart of the quantum system is either quasiregular or chaotic depending on the driving frequency. It is shown that both the quasiregular and the chaotic motion can also support quantum resonances. The relevance of the obtained result to the problem a of crystal electron under simultaneous influence of d.c. and a.c. electric fields is briefly discussed.
\end{abstract}

PACS: 73.20Dx, $73.40 \mathrm{Gk}, 05.45 .+\mathrm{b}$ 
Probing of a quantum system with an electric field is widely used in the experimental physics. If the system has a number of discrete levels (which are associated with classically bounded motion) followed by a continuous spectrum (associated with unbounded motion) then after applying an electric field the discrete energy states become metastable. The decay time of a metastable state is determined by the probability of tunneling through the potential barrier separating the regions of the classically bounded and unbounded motion. Examples are the states of a Rydberg atom or the vibrational state of a dipole molecule in a strong electric field.

The situation is more complicated if a particle should tunnel through many barriers to escape from the regions of bounded motion. A famous example of such a system are the states of an electron in a crystal lattice in an external homogeneous field, which are known as metastable Wannier-Bloch states and Wannier-Stark states [1]. Although the calculation of the decay time for these states is a difficult problem [2], the physics behind the phenomenon is the same - the resonances are supported by the regions of classically bounded motion. The question we address in this letter is, if a quantum resonance states can be supported by "something else" besides a bounded classical motion. To this end we introduce a model, which will be the subject of our study [3]:

$$
H=p^{2} / 2+\cos (\omega t) \cos x+F x \equiv H_{0}+F x .
$$

In Eq. (1) the Hamiltonian $H_{0}$ corresponds to the so-called extended double resonance model, which can show a chaotic diffusion in $x$ for some interval of the driving frequency $\omega$ [4]. The diffusive motion is neither bounded nor ballistic, so the answer to the question - if it can support resonances - is far from obvious.

It should be noted that the Hamiltonian (1) has an explicit periodic dependence on time and, therefore, the notion of energy states is substituted here by the notion of quasienergy states. Below we discuss the metastable quasienergy states of the system for different values of the driving frequency. The only (however important) restriction we impose on $\omega$ is that the period $T_{\omega}=2 \pi / \omega$ should be rational to the Bloch period $T_{B}=\hbar / F[5]$. This 
condition allows us to employ the formalism of quasimomentum, which essentially simplifies the problem. To calculate the metastable states we use the numerical method proposed in Ref. [6]. Using this method one finds the Wannier-Bloch metastable states by diagonalizing the truncated (in the momentum space) operator of the system's evolution over the Bloch period:

$$
\begin{gathered}
W^{(k)} \chi_{l, k}(x)=\exp \left[-i \lambda_{l}(k) T_{B} / \hbar\right] \chi_{l, k}(x), \\
U^{(k)}=\mathrm{e}^{i x} \widehat{\exp }\left\{-\frac{i}{\hbar} \int_{0}^{T_{B}}\left[\frac{(\widehat{p}+\hbar k-F t)^{2}}{2}+V(x, t)\right] \mathrm{d} t\right\} .
\end{gathered}
$$

In Eqs. (2) and (3) $\chi_{l, k}(x)$ is the space-periodic part of the metastable Wannier-Bloch state $\psi_{l, k}(x)=\exp (i k x) \chi_{l, k}(x)$ ( $l$ is the band index, $k$ is the quasimomentum, $\left.-1 / 2<k<1 / 2\right)$, $U^{(k)}$ is one of the possible representations of the $k$-specific system evolution operator over a Bloch period (the hat over the exponent sign denotes time ordering), $W^{(k)}$ is the operator $U^{(k)}$ truncated in momentum space, and $V(x, t)=\cos x \cos (\omega t)$ for the case considered here. We note that the infinite matrix $U_{n, n^{\prime}}^{(k)}=\left\langle n\left|U^{(k)}\right| n^{\prime}\right\rangle$ (here $|n\rangle=(2 \pi)^{-1 / 2} \exp ($ inx $)$ are the basis functions) is unitary, but the truncated $\left(n, n^{\prime} \leq N\right)$ matrix $W_{n, n^{\prime}}^{(k)}$ is not unitary and, therefore, the eigenvalues $\lambda_{l}(k)$ are complex. The key point of the method is that the eigenvectors of the matrix $W_{n, n^{\prime}}^{(k)}$ converge to the metastable Wannier-Bloch states when the dimension of the matrix increases.

We begin with the case of a large driving frequency $\omega \gg \omega_{c r}$ when the dynamics of the system $H_{0}$ is quasiregular [see Fig. 1(a)]. (The critical value $\omega_{c r}$ of the driving frequency for a transition from quasiregular to chaotic dynamics can be estimated by using Chirikov's nonlinear resonance overlap criteria, what corresponds to $\omega_{c r} \approx 1$ [7].) The force $F$ is adjusted to satisfy the resonance condition $2 \pi / \omega=\hbar / F \equiv T$ and $\hbar=0.5$ is used through the paper. For $\omega \gg \omega_{c r}$ the dynamics of the system (1) can be approximately described by the effective Hamiltonian

$$
H^{(+,-)}=\frac{p^{2}}{2}+\frac{1}{2} \cos (x \mp \omega t)+F x
$$


where the plus and minus sign refers to the upper and lower half planes of phase space. By substituting $x^{\prime}=x \mp \omega t$ the Hamiltonian (4) is transformed to the time-independent Hamiltonian $H^{\prime}=p^{2} / 2+0.5 \cos x^{\prime}+F x^{\prime}$ and the known results for Wannier states can be used. Namely, let $\epsilon_{l}$ to be the spectrum of the Wannier-Bloch states (we remind the reader that the energy bands of the Wannier-Bloch states are degenerate, i.e., $\left.\epsilon_{l}(k)=\epsilon_{l}\right)$, then the quasienergy spectrum of the system (4) has the form

$$
\operatorname{Re}\left[\lambda_{l}(k)\right]=\frac{\hbar}{T}\left\{\left(\frac{\omega^{2}}{2}+\operatorname{Re}\left[\epsilon_{l}\right]\right) \frac{T}{\hbar} \mp 2 \pi k\right\}_{\bmod : 2 \pi} \quad, \quad \operatorname{Im}\left[\lambda_{l}(k)\right]=\operatorname{Im}\left[\epsilon_{l}\right] .
$$

The term $2 \pi k$ in Eq. (5) has a simple physical meaning. In fact, similar to the case of a time-independent potential, we can construct from the extended Bloch-like functions $\psi_{l, k}(x)$ a set of the localized states $\Psi_{l, m}(x)=\int \mathrm{d} k \exp (i 2 \pi m k) \psi_{l, k}(x)$. Then the presence of the term $2 \pi k$ denotes that the localized state $\Psi_{l, m}(x)$ moves one lattice period to the right or left per period of the driving frequency.

In view of the above, the spectrum of the system (1) for large $\omega$ should be a symmetric net of parallel lines with positive and negative slopes. The numerical result confirms this prediction (see Fig. 2). We also note that that the spectrum, independent of the value of the driving frequency, possesses the approximate symmetries $\left\langle\lambda_{l}(-k)\right\rangle=\left\langle\lambda_{l}(k)\right\rangle$ and $\left\langle\lambda_{l}(k+1 / 2)\right\rangle=\left\langle\lambda_{l}(k)+\hbar \omega / 2\right\rangle$ (here angle brackets stand for a set of eigenvalues for given $k$ ). These symmetries are a consequence of the exact symmetries of the evolution operator (3) under the transformations $x \rightarrow-x, t \rightarrow-t$ and $x \rightarrow x+\pi, t \rightarrow t+T / 2$. The numerical results depicted in Fig. 2 shows that for $\omega>\omega_{c r}$ the Hamiltonian (1) supports metastable states. This is actually not surprising, because in the frame moving with the velocity $\pm \omega$ the classical trajectory undergoes a bounded oscillation. The main deviation from the case of the "running wave" Hamiltonian (4) is that now the bands $\epsilon_{l}$ in Eq. (5) gain a finite width, which is well seen in the large scale figure of imaginary part of the quasienergy.

We proceed with the case $\omega<\omega_{c r}$ [see Fig. 1(b)]. In this case the phase space of the classical counterpart of the system (1) for $F=0$ consists of two components - a chaotic component in the form of a strip along $x$-axis, and regular component surrounding the 
chaotic region [8]. Since the chaotic and regular components are separated by an invariant curve, a trajectory with an initial condition belonging to the chaotic component stays there forever. For $F \neq 0$ the invariant curve does not exist and a particle always escapes from the chaotic strip to the region of the unbounded regular motion. However, the escape time can be very large. The numerical simulation of the classical dynamics of the system (1) gives an exponential distribution for the escape time $P(t) \sim \exp \left(-t / \tau_{c l}\right)$, where $\tau_{c l}$ tends to infinity as $F$ tends to zero. This result gives us a hint that in the quantum case the Hamiltonian (1) could also support metastable states.

As an example, Fig. 3 shows the real and imaginary part of $\lambda_{l}(k)$ for four quasienergy bands. It is seen that the imaginary part is small, thus, the Hamiltonian (1) does support metastable states. Moreover, we have found that the decay time $\tau_{q u}=\hbar / \operatorname{Im}\left[\lambda_{l}(k)\right]$ of quantum metastable state to be surprisingly large in comparison with the classical decay time $\tau_{c l}$. Figure 4 shows the integrated distribution function $I\left(\tau_{q u}\right)$ for the decay times $\tau_{q u}$ of the quantum states $\psi_{l, k}(x)$ irrespectively to band index and quasimomentum in semilogarithmic scale. It is seen that $\tau_{q u}$ can exceed $\tau_{c l}$ by several orders of magnitude. Thus we encounter a quantum stabilization phenomenon. We also would like to stress the strong resonance-like dependence of the decay rate on quasimomentum $k$. This particular problem requires a further study.

In conclusion, the main result of the paper is the demonstration of the fact that the chaotic unbounded motion is able to support long lived resonances in the quantum case. This result was obtain for the model system (1), which we chose because of its relative simplicity. A more common system corresponds to the Hamiltonian

$$
H=p^{2} / 2+\cos x+F x+\varepsilon x \cos (\omega t)
$$

(a model of a crystal electron subject to d.c. and a.c. electric field). Using the canonical (Krammers-Hennerberger) transformation $p^{\prime}=p+(\varepsilon / \omega) \sin (\omega t), x^{\prime}=x-\left(\varepsilon / \omega^{2}\right) \cos (\omega t)$ it is easy to show that the system (6) is equivalent to a Hamiltonian

$$
H=\frac{p^{2}}{2}+\cos \left[x+\frac{\varepsilon}{\omega^{2}} \cos (\omega t)\right]+F x,
$$


which has a similar structure as the Hamiltonian (1) and also generates chaotic dynamics (see, [9], for example). Thus the obtained result is also valid for the system (6). The detailed discussion of the metastable state of the latter system will be a subject of a forthcoming paper.

This work is supported by DFG through SSP 470. 


\section{REFERENCES}

[1] The Wannier-Bloch and Wannier-Stark states are related to each other by a Fourier transformation [G. H. Wannier, Phys. Rev. 117 (1960) 1366].

[2] J. E. Avron, Ann. Phys. (N.Y.), 143 (1982) 33.

[3] Although the system (1) can be realized in a experiment with optical lattice [see, e.g., M. Raizen, C. Salomon, and Qian Niu, Physics Today, July 1997, 30] we discuss it here from pure academic point of view. A more known model is considered in the concluding remarks of the paper.

[4] A. R. Kolovsky, S. Miayaki, and R. Graham, Phys. Rev. E 49 (1994) 70; M. Glück, A. R. Kolovsky, and H. J. Korsch, Physica D, in press.

[5] This condition is essentially the same as in the paper by Zak [J. Zak, Phys. Rev. Lett. 71 (1993) 2623].

[6] M. Glück, A. R. Kolovsky, and H.J. Korsch, Research workshop "Photo-induced nonlinear dynamics in strong laser fields", Israel, 1998 (unpublished).

[7] See, e.g., A. J. Lichtenberg and M. A. Lieberman, Regular and Chaotic Dynamics (Springer, Berlin, 1983).

[8] Depending on the value of $\omega$, there can also appear additional regular components embedded in the chaotic region. For the chosen value $\omega=0.3$ the chaotic component is uniform, however.

[9] R.Graham, M. Schlautman, and P.Zoller, Phys. Rev. A 45 (1992) R19. 


\section{FIGURES}
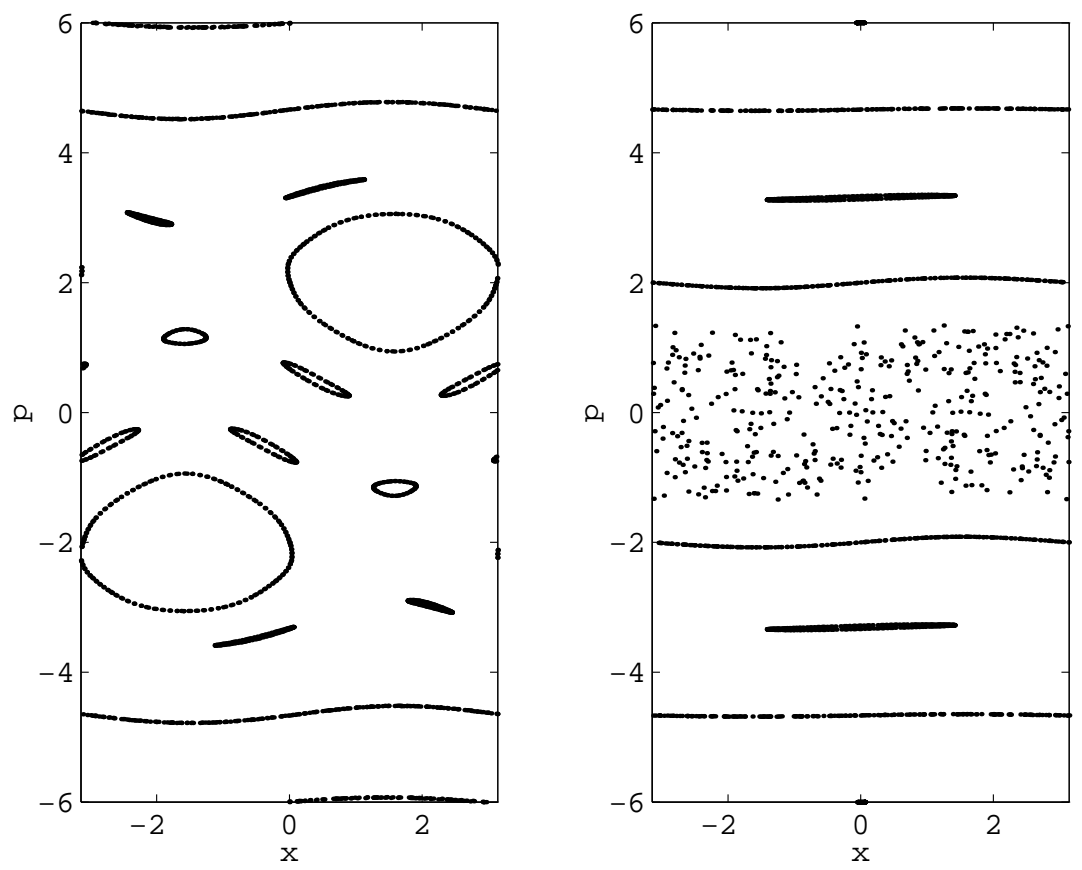

FIG. 1. Phase space portrait of the system (1) for $\omega \approx 2.13>\omega_{c r} \sim 1$ (a) and $\omega=0.3<\omega_{c r}$

(b). Ten different trajectories $p\left(t_{n}\right), x\left(t_{n}\right)_{\bmod : 2 \pi}, t_{n}=n T+T / 4$ are plotted in each case.
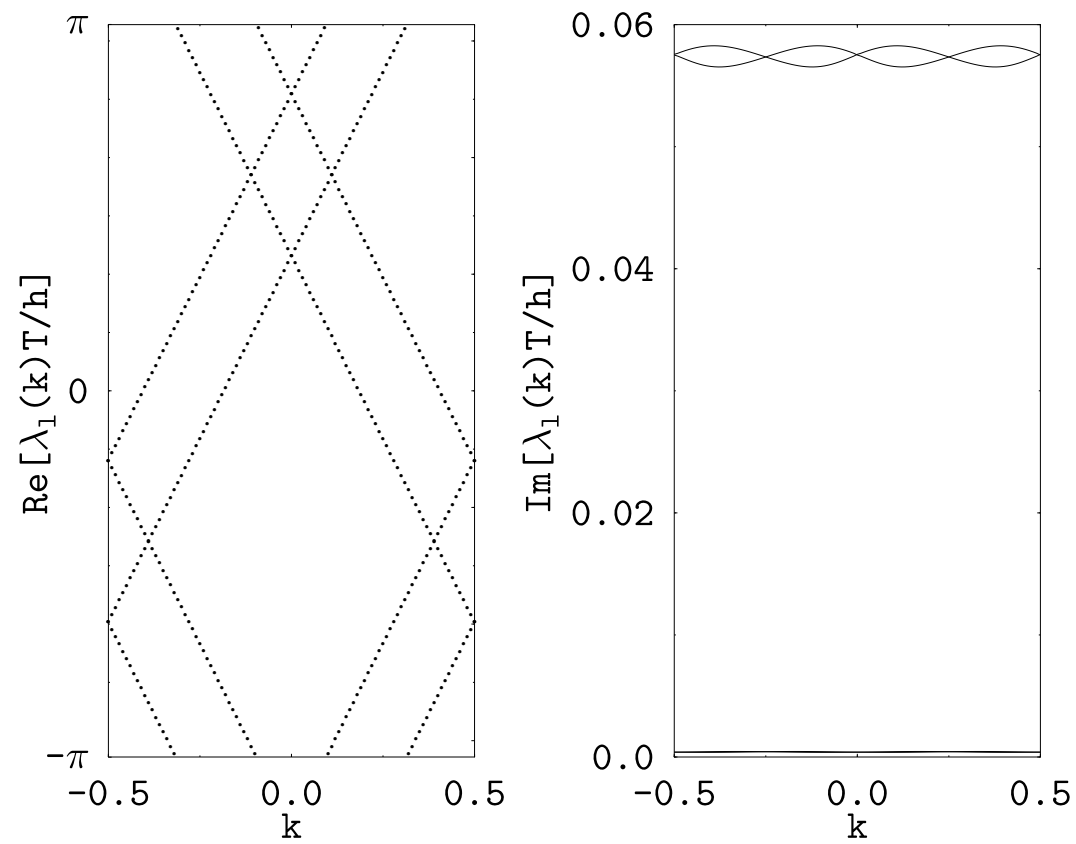

FIG. 2. Real and imaginary parts of the quasienergies corresponding to first four metastable

Wannier-Bloch states of the system (1). The value of the driving frequency is $\omega \approx 2.13$, (scaled Planck constant $\hbar=0.5, F=\hbar \omega / 2 \pi=0.2)$. 

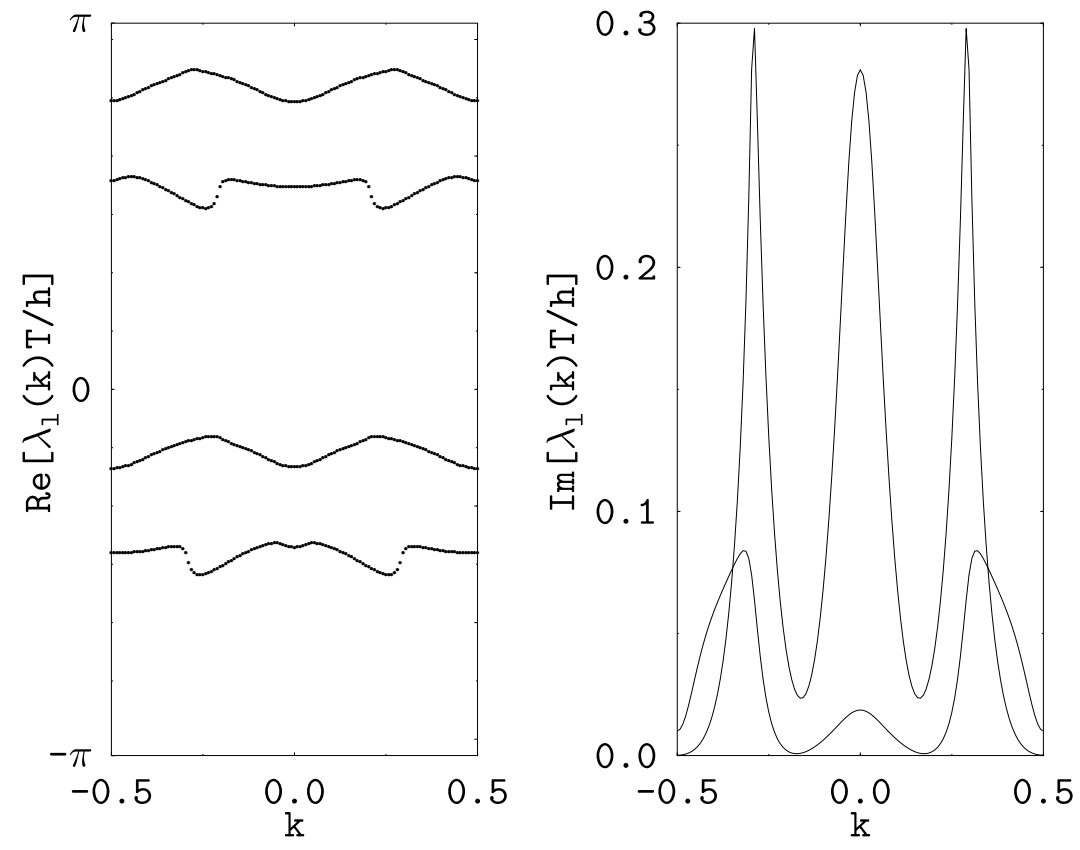

FIG. 3. Same as Fig. 2 but for $\omega=0.3<\omega_{c r}, F=\hbar \omega / 2 \pi \approx 0.02$. (The imaginary part is shown only for two upper bands. For lower bands the curves should be shifted by one half of the Brillouin zone.) 


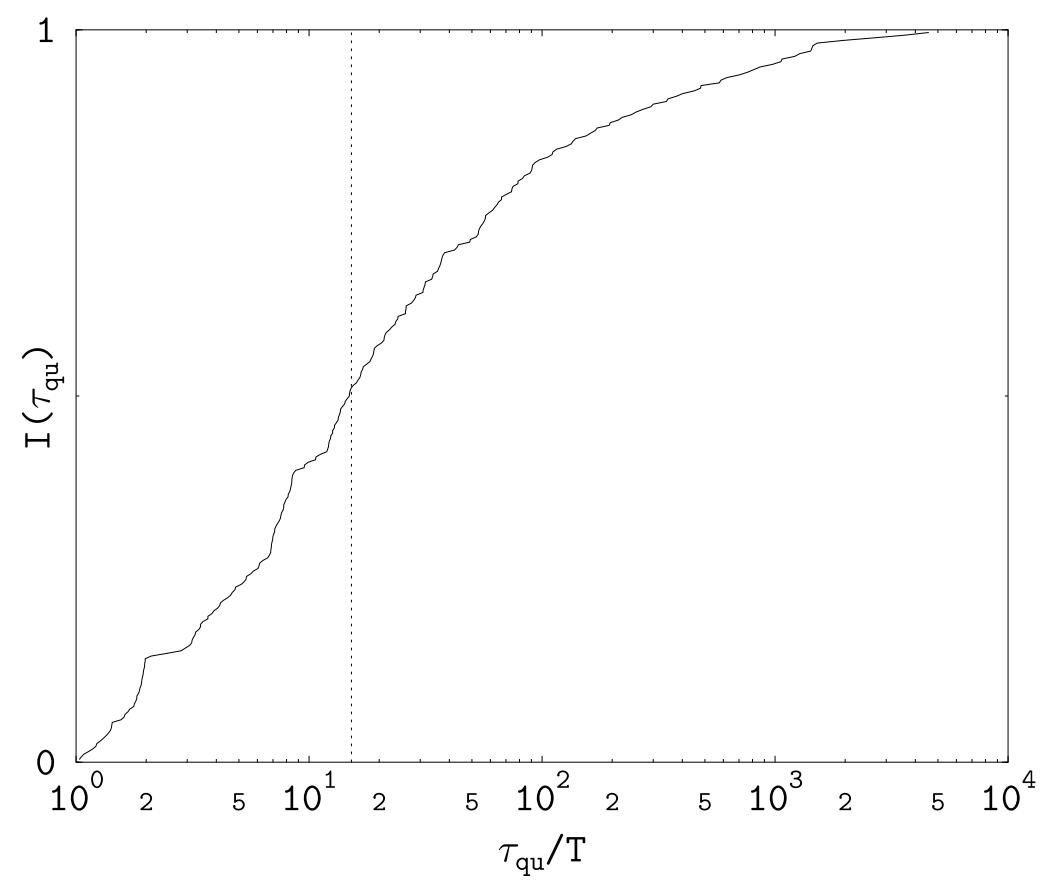

FIG. 4. Integrated distribution function for the decay times $\tau_{q u}=\hbar / \operatorname{Im}\left[\lambda_{l}(k)\right]$ of the quantum metastable states for the parameters of Fig. 3. The vertical line shows the value of the classical decay time $\tau_{c l}=15.2 T$. 